\title{
NRAS Mutation Analysis Was Not Performed
}

National Cancer Institute

\section{Source}

National Cancer Institute. NRAS Mutation Analysis Was Not Performed. NCI Thesaurus.

Code C160478.

An indication that NRAS mutation analysis was not performed during the study. 